\title{
PRODUÇÃO DE PAINÉIS COMPENSADOS COM RESINA FENÓLICA E MELAMÍNICA SEM FORMALDEÍDO
}

\author{
Alexsandro Bayestorff da Cunha ${ }^{1 *}$, Polliana D’Angelo Rios ${ }^{1}$, Ângela Maria Stüpp ${ }^{2}$, \\ Rodrigo Antunes ${ }^{2}$, Martha Andreia Brand ${ }^{1}$ \\ ${ }^{1}$ Universidade do Estado de Santa Catarina, Departamento de Engenharia Florestal, Lajes, Santa Catarina, Brasil - \\ alexsandro.cunha@udesc.br*; polliana.rios@udesc.br; martha.brand@udesc.br \\ ${ }^{2}$ Universidade do Estado de Santa Catarina, Programa de Pós-Graduação em Engenharia Florestal, Lages, Santa Catarina, Brasil - \\ angela.stupp@hotmail.com; eng_rodrigoantunes@hotmail.com
}

Recebido para publicação: 03/06/2015 - Aceito para publicação: 04/10/2016

\begin{abstract}
Resumo
O objetivo desta pesquisa foi avaliar o potencial técnico de duas resinas sem formaldeído para a produção de compensados laminados para ambiente interno e externo não abrigado. Para tanto, as resinas foram comparadas com as tradicionalmente utilizadas pelo setor. O plano experimental foi composto por 05 tratamentos, sendo: com resina fenol sem formaldeído (T1) e com formaldeído (T2), com resina melamina uréia com formaldeído (T3) e sem formaldeído (T4), e com resina uréia formaldeído (T5). Foram produzidos painéis compensados laminados de Pinus taeda com 07 lâminas de $2,7 \mathrm{~mm}$ de espessura, com composição do adesivo e parâmetros de processo conforme recomendado pelos fabricantes das resinas. Observou-se que para ambiente externo não abrigado, a resina melamínica uréia sem formaldeído demonstrou potencial de utilização; já a resina fenólica sem formaldeído (em pó) apresentou resultados pouco expressivos, principalmente na avaliação da qualidade da colagem. Para ambiente interno, a resina melamínica uréia sem formaldeído se apresentou como potencial em termos de propriedades tecnológicas para substituição da uréia formaldeído.

Palavras-chave: Painéis de madeira industrializada; resinas sem formaldeído; propriedades tecnológicas.
\end{abstract}

\begin{abstract}
Production of plywood with phenolic and melamine resin without formaldehyde. The objective of this research was to evaluate the technical potential of two adhesives without formaldehyde for the production of plywood for internal and external not sheltered environment. Therefore, the resins were compared with those traditionally used by the industry. The experimental plan consisted of 05 treatments: phenol resin without formaldehyde (T1) and with formaldehyde (T2), melamine urea with (T3) and without formaldehyde (T4), and urea formaldehyde resin (T5). Plywood laminate panels of Pinus taeda were produced with 07 veneers of $2.7 \mathrm{~mm}$ thickness, with the adhesive composition and process parameters as recommended by the resin manufacturers. It was observed that external environment not sheltered, the urea melamine resin without formaldehyde has shown potential for use; since the phenolic resin without formaldehyde (powder) showed poor results, especially in the bonding quality. For indoors, the resin melamine urea formaldehyde-free introduced himself as a potential in terms of technological properties for replacement of urea formaldehyde. Keywords: Wood panels; resins without formaldehyde; technological properties.
\end{abstract}

\section{INTRODUÇÃO}

O compensado é um painel feito de lâminas de madeira coladas entre si, com as fibras de cada lâmina orientadas perpendicularmente em relação à adjacente. Utiliza-se um número ímpar de lâminas, sendo mais comuns, painéis compostos por 3, 5, 7 ou 9 camadas (TSOUMIS, 1991). Fatores como, espécie de madeira, resina, teor de umidade, quantidade de lenho tardio nas lâminas, pressão e temperatura, utilizados na fabricação dos painéis influenciam diretamente nas propriedades dos painéis (KOLLMANN et al., 1975).

O início da produção industrial desses painéis no mundo se deu por volta do ano de 1910, apresentando um crescimento expressivo até 2007.

Ribaski (2014) afirma que, mesmo o Brasil possuindo condições favoráveis para o melhor desempenho na produção de compensados, é possível verificar que, ao longo dos oito últimos anos, a exportação vem diminuindo, devido a fatores externos e internos. Entre estes fatores, destaca-se, segundo a ABIMCI (2009) a crise mundial de 2008 , tendo em vista que o setor supracitado é altamente depende das exportações.

Os principais fatores limitantes ao desenvolvimento da indústria de compensados no Brasil podem ser resumidos em três pontos principais, como a deficitária estrutura de produção, os elevados custos da matériaprima, principalmente as da Região Norte, e os inexpressivos investimentos tecnológicos realizados nas unidades

FLORESTA, Curitiba, PR, v. 46, n. 4, p. 553 - 560, out. / dez. 2016.

Cunha, A. B. da et al.

ISSN eletrônico 1982-4688

DOI: $10.5380 /$ rf.v46i3.41628 
produtoras (IWAKIRI et al., 2002a).

Entretanto, as vantagens dos painéis de madeira em relação à madeira sólida são perceptíveis já no início do processo de fabricação, pois o aproveitamento expressivo da tora reflete em altos índices de rendimento, quando comparado à conversão em madeira serrada (GONÇALVEZ, 2000). Outras características favoráveis dos painéis em relação à madeira sólida são suas estruturas mais homogêneas e a menor incidência de defeitos, propriedades físico-mecânicas superiores em alguns casos, mais uniformes, maior estabilidade dimensional e maior resistência à biodeterioração (MALONEY, 1996).

O painel de compensado tem múltiplas aplicações. Suas características mecânicas, grandes dimensões e variedades de tipos, adaptáveis a cada uso, constituem os principais atributos para justificar a ampla utilização deste material (MACEDO; ROQUE, 1997). O tipo de uso depende, principalmente, do tipo de resina com que o painel foi fabricado.

Os painéis compensados estruturais são compostos a base de resina fenólica, aplicados principalmente, na construção civil, como forma de concreto, pisos, alma de vigas, cercas, entre outros. Já os compensados não estruturais são compostos a base de resina uréica, utilizados, principalmente, na construção de móveis, constituinte de divisórias, ou outro tipo de objeto que não requer carga excessiva para uso interior.

$\mathrm{Na}$ manufatura de produtos industrializados e reconstituídos de madeira, a resina é um componente fundamental, tendo em vista ser responsável pela ligação entre as peças de madeira e a transferência de tensões geradas na linha de cola durante a sua utilização, além da participação, significativa, na composição de custos de produção (IWAKIRI et al., 2000).

Nas indústrias de compensados e aglomerados, as resinas tradicionalmente utilizadas são as fenólicas e uréicas, sendo estas últimas de baixa resistência à umidade (ROFFAEL, 1982), ambas obtidas pela condensação de formaldeído com fenol ou uréia, respectivamente. Chow (1983) menciona que estas substâncias, por serem oriundas de derivados de petróleo, elevam o custo da resina e, consequentemente, o custo total dos painéis. Após a crise do petróleo, houve, então, uma grande preocupação em substituir estes derivados petroquímicos na indústria de painéis de madeira (CHOW, 1983; PIZZI; MITTAL, 1994). Outro motivo para a substituição de resinas comerciais por resinas naturais é devido ao problema da emissão de substâncias tóxicas (formaldeído) para a atmosfera, como apresentado por Margosian (1990) e Gonçalves et al. (2008).

A fabricação de compensados utilizando resinas que contenham formaldeído em sua composição é um fator relevante tendo em vista que, o painel produzido com esta matéria-prima, libera formol ao longo de toda vida útil. A preocupação com este fator tem crescido e levado à mobilização dos mercados internacionais, onde foram criados limites máximos de emissão de formol pelo painel e a criação de barreiras técnicas para exportação, como o CARB (California Environmental Protection) para o mercado americano e o RECH (Register, Evaluation, Authorization, Chemicals) para o mercado europeu.

Desta forma, o objetivo desta pesquisa foi avaliar o potencial técnico de duas resinas, sem formaldeído, para a produção de compensados laminados destinados a uso em ambiente interno e externo não abrigado. Para tanto, as resinas foram comparadas com as tradicionalmente utilizadas pelo setor.

\section{MATERIAL E MÉTODOS}

Foram utilizadas nesta pesquisa lâminas de Pinus taeda com dimensões de $560 \times 560 \times 2,7 \mathrm{~mm}$, retiradas do processo industrial da empresa Compensados Nereu Rodrigues de Correia Pinto, Santa Catarina, resina fenólica com e sem formaldeído, resina melamina uréia com e sem formaldeído, e resina uréia formaldeído.

Segundo informações da empresa, as resinas isentas de formaldeído livre são obtidas pelo processo de fabricação fenol-formol (para resina fenólica em pó) e pelo processo de fabricação melamina-formol (para resina melamina). Ambas as resinas são submetidas ao processo de desidratação (retirada de água e formol livre) por meio de exaustores, onde libera-se uma quantidade considerável de formol em um processo controlado. A resina fenol-formol é utilizada na forma de pó, contendo característica adesiva através da fusão em alta temperatura, enquanto que a resina melamina-formol passa pelo processo de reidratação, para ser utilizada na forma líquida com propriedade adesiva em alta temperatura. Todo formol contido no ato da fabricação das resinas, polimeriza e formam outros componentes poliméricos. Resumidamente, o formol está presente na reação química que a envolve, porém, não está presente na sua forma desidratada ou em resinas que passam por este processo. Assim, o produto resultante de sua utilização é caracterizado como formol free.

$\mathrm{O}$ delineamento experimental envolveu cinco tratamentos, os quais foram compostos pelos diferentes tipos de resina (Tabela 1). Para a produção dos quatro painéis compensados por tratamento, as lâminas foram secas em estufa até teor de umidade médio de $10 \%$. Os painéis foram produzidos com sete lâminas, com formulações de batida de cola, gramatura e ciclo de prensagem conforme recomendação dos fabricantes das resinas (Tabela 2). 
Tabela 1. Plano experimental.

Table 1. Experimental chart.

\begin{tabular}{lll}
\hline Tratamento & Resina & Formulação do adesivo \\
\hline T1 & Fenólica sem formaldeído & 100 partes resina em pó \\
T2 & Fenólica com formaldeído & 100 partes de resina, 12 extensor, 12 água \\
T3 & Melamina uréia com formaldeído & 100 partes resina, 20 extensor, 10 água \\
T4 & Melamina uréia sem formaldeído & 100 resina líquida \\
T5 & Uréia com formaldeído & 100 parte de resina, 20 extensor, 10 água \\
\hline
\end{tabular}

Tabela 2. Gramatura do adesivo e parâmetros do ciclo de prensagem.

Table 2. Amount of adhesive and parameters of the press cycle.

\begin{tabular}{lllll}
\hline Tratamento & Gramatura $\left(\mathbf{g} / \mathbf{m}^{\mathbf{2}}\right)$ & Pressão $\left(\mathbf{k g f} / \mathbf{c m}^{\mathbf{2}}\right)$ & Temperatura $\left(\mathbf{~}^{\mathbf{0}} \mathbf{C}\right)$ & Tempo $(\mathbf{m i n})$ \\
\hline T1 & 60 & 14 & 150 & 18 \\
T2 & 400 & 10 & 140 & 18 \\
T3 & 360 & 10 & 130 & 18 \\
T4 & 360 & 10 & 130 & 18 \\
T5 & 360 & 10 & 110 & 18 \\
\hline
\end{tabular}

Após a prensagem, os painéis foram esquadrejados e acondicionados na câmara climática à temperatura de $20 \pm 3{ }^{\circ} \mathrm{C}$ e umidade relativa de $65 \pm 5 \%$ até sua estabilização, sendo posteriormente cortados em corpos de prova de acordo com as normas de referência utilizadas nos ensaios.

A determinação da massa específica e do teor de umidade dos painéis, de todos os tratamentos foi realizada com base nos procedimentos da EN 323 (2000) e da EN 322 (2000), respectivamente, enquanto que a determinação do módulo de ruptura (MOR) e elasticidade (MOE) foi realizada por meio da EN 310 (2000). Entretanto, como as resinas utilizadas possuem diferentes condições de uso (fenólica: externo, melamínica: intermediário e uréia: interno), o ensaio de cisalhamento na linha de cola foi diferenciado, conforme prescreve a EN 314-1 (1996) e a EN 314-2 (1996).

A resina fenólica e a melamínica foram comparadas por meio de dois pré-tratamentos, (1) imersão durante 24 horas em água a temperatura a $20+3{ }^{\circ} \mathrm{C}$ e (2) imersão durante 4 horas em água em ebulição, seguida de secagem em estufa durante 20 horas a $60 \pm 3{ }^{\circ} \mathrm{C}$, seguida de imersão em água em ebulição durante 4 horas, e seguida de um resfriamento em água a $20 \pm 3{ }^{\circ} \mathrm{C}$ durante uma hora. Já a melamínica e a uréia foram comparadas somente por meio do pré-tratamento (1).

O modelo experimental utilizado para a análise estatística foi delineamento inteiramente casualizado (DIC). Cabe salientar que os tratamentos T1 e T2 foram comparados somente com os tratamentos T3 e T4, enquanto que o tratamento T5 foi comparado somente com o T3 e T4; esta análise diferenciada se deve ao uso dos painéis laminados: resina fenólica para ambiente externo, resina melamínica para ambiente intermediário e resina uréica para ambiente interno. A distribuição da normalidade dos dados foi avaliada por meio do teste de Shapiro Wilk, enquanto a homogeneidade das variâncias foi verificada pela aplicação do teste de Bartlett. Com os pressupostos atendidos, cada variável resposta foi submetida à análise de variância. Havendo diferença entre os resultados, realizou-se o teste de médias de Scott-Knott a 95\% de probabilidade.

\section{RESULTADOS E DISCUSSÃO}

\section{Massa específica e teor de umidade}

Os valores médios de massa específica e teor de umidade dos painéis estão apresentados na tabela 3 com seus respectivos coeficientes de variação.

Tabela 3. Valores médios de massa específica e teor de umidade.

Table 3. Medium values of specific gravity and moisture content.

\begin{tabular}{lll}
\hline Tratamento & Massa específica $\left(\mathbf{k g} / \mathbf{m}^{\mathbf{3}}\right)$ & Teor de umidade $(\%)$ \\
\hline T1 - F s/f & $508_{(06,17)} \mathrm{A}$ & $12_{(02,78)} \mathrm{A}$ \\
T2 - F c/f & $523_{(06,49)} \mathrm{A}$ & $10_{(03,11)} \mathrm{A}$ \\
T3 - MU c/f & $512_{(10,34)} \mathrm{Aa}$ & $11_{(06,71)} \mathrm{Aa}$ \\
T4 - MU s/f & $520_{(09,52)} \mathrm{Aa}$ & $10_{(12,79)} \mathrm{Aa}$ \\
T5 - U c/f & $526_{(10,51)} \mathrm{a}$ & $10_{(11,68)} \mathrm{a}$ \\
\hline
\end{tabular}

Legenda: F: fenólica; MU: melamina uréia; U: ureia, s/f: sem formaldeído; c/f: com formaldeído; subscrito: coeficiente de variação (\%); letras maiúsculas na mesma coluna não diferenciam estatisticamente entre si; letras minúsculas na mesma coluna não diferenciam entre si, ambos pelo teste de Scott Knott a 95\% de probabilidade.

FLORESTA, Curitiba, PR, v. 46, n. 4, p. 553 - 560, out. / dez. 2016.

Cunha, A. B. da et al.

ISSN eletrônico 1982-4688

DOI: $10.5380 /$ rf.v46i3.41628 
Pode-se observar que o intervalo de variação está entre 508 e $523 \mathrm{~kg} / \mathrm{m}^{3}$ para a primeira variável e entre 10 e $13 \%$ para a segunda. Cabe destacar que não houve diferença estatística entre os tratamentos para as duas variáveis analisadas.

Os valores encontrados para massa específica de todos os tratamentos estão dentro do intervalo estabelecido pela ABIMCI (2007) para painéis de Pinus spp com $18 \mathrm{~mm}$ de espessura, resina fenólica e com qualidade $\mathrm{C}+/ \mathrm{C}$, que é de 496 a $620 \mathrm{~kg} / \mathrm{m}^{3}$. Da mesma forma, o teor de umidade deve estar entre 10 e $12 \%$ conforme a norma da associação supracitada.

Tomando-se como referência o estudo realizado por Cunha et al. (2005), em empresas de compensados laminados fenólicos de Pinus taeda da região sul do Paraná e norte de Santa Catarina, as quais utilizavam em seus produtos, características semelhantes as utilizadas no presente estudo, em termos de espessura de lâmina e de painel, gramatura e ciclo de prensagem, tem-se que, das oito empresas analisadas, cinco apresentam painéis com massa específica entre 521 e $535 \mathrm{~kg} / \mathrm{m}^{3}$ e três acima de $592 \mathrm{~kg} / \mathrm{m}^{3}$. Em relação ao teor de umidade, o intervalo de variação entre os painéis das empresas foi de $8,30 \%$ a $11,08 \%$.

Acredita-se que, as diferenças dos valores de massa específica encontradas no presente estudo, e nos painéis das empresas do estudo de Cunha et al. (2005), se deva a qualidade das lâminas, no que se refere à idade das árvores utilizadas para a produção dos painéis, posição de retirada da lâmina da tora (próximo à medula, porção intermediária ou próximo a casca; e ainda ao longo do fuste), assim, salienta-se que as diferentes resinas não contribuíram, ou pouco contribuíram, para a diferença, tanto que, entre elas (Tabela 3), não foi encontrada diferença estatística.

Quanto ao teor de umidade apresentado na tabela 3, nota-se que não houve diferença estatística entre os painéis produzidos no laboratório, tanto para ambiente externo quanto para ambiente interno.

\section{Flexão estática paralela e perpendicular}

Nos valores médios de módulo de elasticidade paralelo e perpendicular apresentados na tabela 4, observa-se que não houve diferença estatística entre os tratamentos.

Tabela 4. Valores médios para módulo de elasticidade e módulo de ruptura.

Table 4. Medium values for modules of rupture and elasticity.

\begin{tabular}{lllll}
\hline \multirow{2}{*}{ Tratamento } & \multicolumn{3}{c}{ MOE (MPa) } & \multicolumn{2}{c}{ MOR (MPa) } \\
\cline { 2 - 5 } & Paralelo & Perpendicular & Paralelo & Perpendicular \\
\hline T1 - F s/f & $4102_{(21,53)} \mathrm{A}$ & $1838_{(19,78)} \mathrm{A}$ & $28,16_{(24,77)} \mathrm{A}$ & $14,87_{(30,47)} \mathrm{A}$ \\
T2 - F c/f & $3657_{(22,70)} \mathrm{A}$ & $1729_{(19,69)} \mathrm{A}$ & $31,35_{(17,78)} \mathrm{A}$ & $25,03_{(17,41)} \mathrm{A}$ \\
T3 - MU c/f & $4024_{(29,57)} \mathrm{Aa}$ & $1761_{(21,03)} \mathrm{Aa}$ & $40,04_{(25,01)} \mathrm{Aa}$ & $24,18_{(29,98)} \mathrm{Aa}$ \\
T4 - MU s/f & $4275_{(12,54)} \mathrm{Aa}$ & $1404_{(16,81)} \mathrm{Aa}$ & $36,97_{(10,07)} \mathrm{Aa}$ & $19,86_{(30,23)} \mathrm{Aa}$ \\
T5 - U c/f & $4450_{(31,18)} \mathrm{a}$ & $1782_{(14,98)} \mathrm{a}$ & $34,54_{(25,60)} \mathrm{a}$ & $20,73_{(10,49)} \mathrm{a}$ \\
\hline
\end{tabular}

Legenda: F: fenólica; MU: melamina uréia; U: ureia, s/f: sem formaldeído; c/f: com formaldeído; subscrito: coeficiente de variação (\%); letras maiúsculas na mesma coluna não diferenciam estatisticamente entre si; letras minúsculas na mesma coluna não diferenciam entre si, ambos pelo teste de Scott Knott a 95\% de probabilidade.

A ausência de diferença estatística para MOE e MOR entre os tratamentos (Tabela 4) foi evidenciada quando se avalia de forma isolada as resinas destinadas a uso exterior não abrigado (FF e MU), e quando se analisa as resinas para uso interno (UF e MU), demonstrando que o tipo de resina não interferiu nas propriedades de rigidez e resistência dos painéis.

Segundo a ABIMCI (2007), os painéis de Pinus spp produzidos com resina fenólica e parâmetros de processo compatíveis ao do presente estudo devem apresentar valores de MOE paralelo entre 3274,61 e 6413,12 MPa, MOE perpendicular entre 2167,82 e 4675,40 MPa, MOR paralelo entre 28,05 e 54,88 MPa e MOR perpendicular entre 22,08 e 47,29 MPa. Desta forma, é possível observar que entre as resinas de uso exterior não abrigado (FF e MU), somente os valores de MOE e MOR paralelo atenderam ao parâmetro estabelecido pela associação, enquanto que para MOE e MOR perpendicular os tratamentos compostos por painéis com resina sem formaldeído ficaram abaixo do mínimo estabelecido.

Bortoletto Júnior e Garcia (2004) analisaram painéis compensados de Pinus spp com 11 lâminas, colados com resina fenol formaldeído e chegaram a valores de MOE paralelo e perpendicular de $5932 \mathrm{MPa}$ e $3966 \mathrm{MPa}$, respectivamente, e valores de MOR paralelo e perpendicular de $40 \mathrm{MPa}$ e $36 \mathrm{MPa}$, ou seja, superiores ao presente estudo e, também, com uma diferença numérica entre o sentido perpendicular e paralelo menos expressiva. Assim, os autores atribuem que painéis com maior número de lâminas proporcionam uma resistência mais uniforme entre as direções.

Em relação ao estudo de Cunha et al. (2005), tem-se valores entre 2986 e $8441 \mathrm{MPa}$ para MOE 
paralelo, entre 2439 e $3933 \mathrm{MPa}$ para MOE perpendicular, entre 29,48 e 55,89 MPa para MOR paralelo e entre 22,55 e 39,53 MPa, demonstrando, desta forma, uma grande variação em função da qualidade da madeira utilizada e não do tipo de resina que é a mesma, fenol formaldeído, pois as composições e gramaturas foram semelhantes a da presente pesquisa.

Para a resina de uso interior (UF), Iwakiri et al. (2002b) encontraram para painéis compensados de lâminas de Pinus taeda com 20 anos de idade e em 3 formulações de adesivo, valores de MOE entre 8649,81 e 9486,55 MPa e de MOR entre 65,31 e 73,29 MPa, os quais são bem superiores as médias encontradas nesta pesquisa, para os painéis colados com melamina uréia e uréia formaldeído. Entretanto, outro estudo de Iwakiri et al. (2001) com painéis compensados utilizando espécies de pinus tropicais e adesivo a base de resina uréia formaldeído, encontrou valores de MOE e MOR para Pinus caribeae, Pinus chiapensis, Pinus maximinoi, Pinus oocarpa e Pinus taeda: 3877,34 e 34,87 MPa, 4223,91 e 38,28 MPa, 6838 e 39,49 MPa, 6158,93 e 33,11 MPa, e 4330,27 e 32,01 MPa, respectivamente, os quais são semelhantes ao do presente estudo, com exceção aos valores de MOE dos painéis da terceira e quarta espécies.

\section{Cisalhamento na linha de cola}

Os valores médios de tensão e porcentagem de falha na madeira, apresentados na tabela 5, são resultantes dos ensaios em três situações (pré-tratamentos), conforme prescrito na norma adotada.

Tabela 5. Valores médios de cisalhamento na linha de cola.

Table 5. Medium values for bonding line shear.

\begin{tabular}{lllllll}
\hline \multirow{2}{*}{ Tratamento } & \multicolumn{2}{c}{ Ensaio seco } & \multicolumn{2}{c}{ Ensaio úmido } & \multicolumn{2}{c}{ Ensaio ciclo } \\
\cline { 2 - 7 } & Tensão (MPa) & FM (\%) & Tensão (MPa) & FM (\%) & Tensão (MPa) & FM (\%) \\
\hline T1 - F s/f. & $1,21_{(22,07)} \mathrm{D}$ & $14_{(26,59)} \mathrm{C}$ & $0,36_{(17,47)} \mathrm{C}$ & $00_{(00,00)} \mathrm{C}$ & $0,00_{(00,00)} \mathrm{B}$ & $00_{(00,00)} \mathrm{B}$ \\
T2 - F c/f. & $2,67_{(22,36)} \mathrm{A}$ & $45_{(19,56)} \mathrm{A}$ & $1,32_{(23,38)} \mathrm{A}$ & $32_{(29,56)} \mathrm{A}$ & $1,34_{(22,86)} \mathrm{A}$ & $18_{(28,95)} \mathrm{A}$ \\
$\mathrm{T} 3-\mathrm{MU}$ c/f. & $1,80_{(18,20)} \mathrm{Cb}$ & $29_{(24,92)} \mathrm{Ba}$ & $0,87_{(22,69)} \mathrm{Bb}$ & $11_{(28,83)} \mathrm{Bc}$ & $0,00_{(00,00)} \mathrm{B}$ & $00_{(00,00)} \mathrm{B}$ \\
$\mathrm{T} 4-\mathrm{MU} \mathrm{s} / \mathrm{f}$. & $2,13_{(22,08)} \mathrm{Ba}$ & $23_{(16,17)} \mathrm{Ba}$ & $1,60_{(20,95)} \mathrm{Aa}$ & $30_{(24,26)} \mathrm{Aa}$ & $1,09_{(24,97)} \mathrm{A}$ & $14_{(23,20)} \mathrm{A}$ \\
T5 - U c/f. & $1,84_{(24,89)} \mathrm{b}$ & $26_{(28,96)} \mathrm{a}$ & $0,93_{(25,91)} \mathrm{b}$ & $23_{(23,59)} \mathrm{b}$ & ---- & -- \\
\hline
\end{tabular}

Legenda: F: fenólica; MU: melamina uréia; U: ureia, s/f: sem formaldeído; c/f: com formaldeído, FM: falha na madeira; subscrito: coeficiente de variação (\%); letras maiúsculas na mesma coluna não diferenciam estatisticamente entre si; letras minúsculas na mesma coluna não diferenciam entre si, ambos pelo teste de Scott Knott a 95\% de probabilidade.

No que se refere à condição seca, os valores médios obtidos no ensaio demonstram uma grande heterogeneidade entre os tratamentos que utilizaram resina para uso exterior (fenólica e melamínica), o que é visualizado por meio dos números apresentados e pela presença da significância estatística. $\mathrm{O}$ tratamento que apresentou o melhor desempenho foi o T2, com fenol formaldeído, e o pior, o T1, com fenol e sem formaldeído.

Iwakiri et al. (2002a) encontraram, para painéis compensados de Pinus taeda e Pinus oocarpa com resina formaldeído, valores de tensão entre 1,88 e 2,81 MPa para a primeira espécie e, entre 1,92 e 2,61 MPa, para a segunda. Confrontando com os valores da tabela 5, denota-se que os únicos tratamentos equivalentes são o T2 (fenol formaldeído) e o T4 (melamína uréia sem formaldeído), enquanto que os demais apresentam valores inferiores. Em relação à norma britânica B.S.1203 (1954), que estabelece parâmetro mínimo para condição seca de 1,8 MPa, constata-se o enquadramento de três tratamentos, T2 (fenol formaldeído), T3 e T4 (melamina uréia com e sem formaldeído).

Já entre as resinas de ambiente interno (melamínica e uréica), na condição seca, observa-se a superioridade do tratamento T4 (melamínica sem formaldeído) em relação ao T3 (melaminíca com formaldeído) e ao T5 (uréia formaldeído), na variável tensão e uma similaridade na porcentagem de falha na madeira. Nesta condição, Iwakiri et al. (2002b) encontraram intervalo de tensão para painéis compensados uréicos de Pinus taeda, entre 1,06 e 1,57 MPa, o que é inferior aos três tratamentos utilizados no presente estudo.

Para a condição úmida (24 horas em água), os melhores tratamentos, no que tange as resinas para uso externo, foram o T2 e T4, que são compostos por resina fenólica com formaldeído e melamínica sem formaldeído, sendo que a resina em pó (fenólica sem formaldeído, T1) apresentou o valor menos expressivo para tensão e nulo para porcentagem de falhas na madeira. Cunha et al. (2005) encontraram valores entre 0,92 e 1,59 MPa para os painéis fenólicos das empresas da região sul do Brasil, o que engloba três tratamentos do presente estudo, T2 (fenol formaldeído), T3 e T4 (melamínica com e sem formaldeído). Tomando-se como referência a norma EN 314 (1996), observa-se que todos os tratamentos com resina para ambiente externo não abrigado estão de acordo com os parâmetros da norma, com exceção do tratamento T1, que não atendeu ao requisito mínimo. 
Ainda na condição úmida, alterando o uso da resina para ambiente interno (melamínica e uréica), evidencia-se que o melhor tratamento foi o T4 (melamina sem formaldeído) e, também, que o mesmo foi o único a atender o parâmetro mínimo da EN 314 (1996), que especifica tensão maior que 1 MPa sem exigência de valor mínimo para porcentagem de falha na madeira.

No pré-tratamento mais drástico, com ciclo de fervura e secagem, verifica-se, por meio da tabela 5, que os tratamentos T1 (fenólica sem formaldeído) e T3 (melamina uréia com formaldeído) apresentaram valores nulos de tensão e porcentagem de falha na madeira, o que foi evidenciado ainda durante a aplicação do prétratamento. Já as outras duas resinas para ambiente externo, nota-se que apresentaram valores médios compatíveis com a norma EN 314 (1996), mesmo apresentando uma baixa porcentagem de falha na madeira.

Em relação a outros estudos desenvolvidos para painéis fenólicos, com aplicação do pré-tratamento supracitado (ciclo de fervura e secagem), pode-se citar Cunha et al. (2005), que encontraram para as empresas da região sul do Brasil, valores de 0,69 a 1,40 MPa; Iwakiri et al. (2002a), que determinaram intervalo entre 0,94 a 1,26 MPa para painéis compensados de Pinus taeda produzidos com resina fenol-formaldeído; Iwakiri et al. (2009) que encontraram para compensados fenólicos com cinco espécies de pinus tropicais (P. chiapensis, $P$. caribeae var. hondurensis, $P$. maximinoi, $P$. oocarpa e P. tecunumannii), valores entre 0,88 e 1,42 MPa e, para Pinus taeda, 1,32 MPa; e Prata (2006), que definiu intervalo entre 0,78 e 1,33 MPa para painéis fenólicos $\mathrm{cm}$ Pinus spp.

\section{CONCLUSÕES}

- As diferentes resinas utilizadas para a produção dos painéis compensados não influenciaram nos valores de massa específica e teor de umidade, demonstrando que houve homogeneidade da matéria-prima utilizada e do processo de produção empregado.

- Para ambiente externo, não abrigado, pode-se concluir que, a resina melamínica uréia sem formaldeído demonstrou potencial de utilização, tendo em vista que apresentou valores equivalentes a resina tradicionalmente utilizada para este fim, fenol formaldeído. Todavia, a resina fenólica sem formaldeído (em pó) apresentou resultados pouco expressivos, principalmente, no que tange a qualidade da colagem.

- Em relação ao ambiente interno, a resina melamínica uréia sem formaldeído se apresentou como potencial para substituição da uréia formaldeído, necessitando de uma análise econômica para determinar a viabilidade de utilização em nível industrial.

\section{AGRADECIMENTOS}

As empresas Compensados Nereu Rodrigues Ltda, Royal Plas e Quimisa S.A. e a Fundação de Amparo à Pesquisa e Inovação do Estado de Santa Catarina (FAPESC).

\section{REFERÊNCIAS}

\section{ASSOCIAÇÃO BRASILEIRA DA INDÚSTRIA DA MADEIRA PROCESSADA MECANICAMENTE (ABIMCI). Estudo setorial. Curitiba, 2007.}

Estudo Setorial 2009, ano base 2008. Catálogo Técnico Curitiba, 2009.

BORTOLETTO JÚNIOR, G.; GARCIA, J. N. Propriedades de resistência e rigidez à flexão estática de painéis OSB e compensados. Árvore, Viçosa, v. 28, n. 4, p. 563-570, 2004.

BRITISH STANDARD INSTITUTION. Specification for synthetic resin adhesives for plywood. B.S. 1203. London, 1954. $11 \mathrm{p}$.

CHOW, S. Adhesive developments in Forest products. Wood Science and Technology. Springer-Verlag: New York, v. 17, p. 01-11, 1983.

CUNHA, A.; WALTER, A. P.; MAIDEL, R.; LIMA, R. L.; LINZMEIER, D. M.; SÔNEGO JÚNIOR, E. Caracterização tecnológica dos painéis compensados das empresas da região sul do Paraná e norte de Santa Catarina. Relatório Técnico, 2005.

EN 310:2000 Painéis derivados de Madeira - Determinação do módulo de elasticidade European Stardard (versão portuguesa), Bruxelas 2000.

EN 314-1:1996 Colagem do Compensado de Madeira - Qualidade - Parte1: Método de Teste. European Stardard (versão portuguesa), Bruxelas 1996. 
EN 314-2:1996 Colagem do Compensado de Madeira - Qualidade - Parte 2: Exigências de Teste. European Stardard (versão portuguesa), Bruxelas 1996.

EN 322:2000 Painéis derivados de Madeira - Determinação do teor de umidade. European Stardard (versão portuguesa), Bruxelas 2000.

EN 323:2000 Painéis derivados de Madeira - Determinação da massa específica. European Stardard (versão portuguesa), Bruxelas 2000.

GONÇALVES, M. T. T. Processamento da madeira. Document Center Xerox - USC, Bauru, 2000, p. 242.

IWAKIRI, S.; OLANDOSKI, D. P.; LEONHARDT, G.; BRAND, M. A. Produção de chapas de madeira compensada de cinco espécies de pinus tropicais. Ciência Florestal, Santa Maria, v. 11, n. 2, p. 71-77, 2001.

IWAKIRI, S.; NIELSEN, I. R.; ALBERTI, R. A. R. Avaliação da influência de diferentes composições de lâminas em compensados estruturais de Pinus elliottii e Eucalyptus saligna. Cerne, Lavras, v. 6, n. 2, p. 19-24, 2000.

IWAKIRI, S.; DEL MENEZZI, C. H. S.; LAROCA, C.; VENSON, I.; MATOSKI, S. S. Produção de compensados de Pinus taeda e Pinus oocarpa com resina fenol-formaldeído. Cerne, Lavras, v. 8, n. 2, p. 92-97, $2002 \mathrm{a}$.

IWAKIRI, S.; SILVA, J. C.; SILVA, J. R. M. da; AlVES, C. R. E.; PUEHRINGER, C. A produção de compensados de Pinus taeda L. e Pinus oocarpa Schiede com diferentes formulações de adesivo uréia formaldeído. Árvore, Viçosa, v. 26, n. 3, 2002 b.

IWAKIRI, S.; MATOS, J. L. M.; LIMA, A. J. M.; FERREIRA, E. S.; BATISTA, D. C.; ROMÃO, S. A. A. Produção de painéis compensados de pinus tropicais colados com resina fenol-formaldeído. Floresta, Curitiba, v. 39, n. 3, p. 669-673, 2009.

IWAKIRI, S.; MANHIÇA, A. A.; RAMIREZ, M. G. L.; MATOS, J. L. M.; BONDUELLE, G. M.; PRATA, J. G. Avaliação da qualidade de painéis compensados de Pinus maximinoi, Pinus oocarpa e Pinus tecunumannii com diferentes composições estruturais. Ciência Florestal, Santa Maria, v. 22, n. 2, p. 315-321, 2012.

KOLLMANn, F. F. P.; KUENZI, E. W.; STAMM, A. J. Principles of wood science and technology. Wood based materials. Springer-Verlag: Berlin, 1975, v. 2, p. 154-283.

MACEDO, A. R. P.; ROQUE, C. A. L. Painéis de Madeira. BNDES Setorial. Rio de Janeiro, n. 6, p. 117-132, 1997.

MALONEY, T. M. The family of wood composite materials. Forest Products Journal, Madison, v. 2, n. 46, p. 19-26, 1996.

MARGOSIAN, R. Initial formaldehyde emission levels for particleboard manufactured in the United States. Forest Products Journal, Madison, v. 40, n. 6, p. 19-20, 1990.

PIZZI, A.; MITTAL, K. L. Handbook of Adhesive Technology, Marcel Dekker, Chapter 19, 1994, p. $347-358$.

PRATA, J. G. Desempenho de um sistema de qualidade em uma fábrica de painéis compensados. 118 p. Dissertação (Mestrado em Engenharia Florestal) - Curso de Pós-Graduação em Engenharia Florestal, Setor de Ciências Agrárias, Universidade Federal do Paraná. Curitiba, 2006.

RIBASKI, N. G.; HILLIG, É.; MIRANDA, G. M. A crise internacional x produção de compensados do estado do Paraná. Conhecimento Interativo, v. 8, p. 123-142, 2014.

ROFFAEL, E. Die Formaldehydabgabe von Spanplatten und anderen Holzwerkstoffen. DRW-Verlag, Stuttgart, 154 p. 1982.

TSOUMIS, G. Science and technology of wood: structure, properties, utilization. New York: Chapman \& Hall, 1991, 494 p. 